\title{
Corrigendum: Hypocoagulability and Platelet Dysfunction Are Exacerbated by Synthetic Colloids in a Canine Hemorrhagic Shock Model
}

\author{
Corrin J. Boyd*, Melissa A. Claus, Anthea L. Raisis, Giselle Hosgood, Claire R. Sharp and \\ Lisa Smart
}

School of Veterinary and Life Sciences, College of Veterinary Medicine, Murdoch University, Perth, WA, Australia

Keywords: hydroxyethyl starch, succinylated gelatin, crystalloid, fresh whole blood, platelet closure time, PFA-100, rotational thromboelastometry (ROTEM), viscoelastic coagulation tests

\section{A Corrigendum on}

Hypocoagulability and Platelet Dysfunction Are Exacerbated by Synthetic Colloids in a Canine Hemorrhagic Shock Model

by Boyd, C. J., Claus, M. A., Raisis, A. L., Hosgood, G., Sharp, C. R., and Smart, L. (2018). Front. Vet. Sci. 5:279. doi: 10.3389/fvets.2018.00279

In the original article, there was a mistake in Table 2 as published. The EXTEM CFT baseline mean (95\% confidence interval) for the HES group was slightly incorrect due to a data entry error. The corrected Table 2 appears below.

In the original article, there was a mistake in Table 5 as published. The EXTEM CFT baseline

Edited and reviewed by:

Katja-Nicole Adamik,

University of Bern, Switzerland

*Correspondence:

Corrin J. Boyd

c.boyd@murdoch.edu.au

Specialty section:

This article was submitted to Veterinary Emergency and Critical

Care Medicine,

a section of the journal

Frontiers in Veterinary Science

Received: 29 June 2020

Accepted: 06 August 2020

Published: 08 September 2020

Citation:

Boyd CJ, Claus MA, Raisis AL, Hosgood G, Sharp CR and Smart $L$

(2020) Corrigendum: Hypocoagulability and Platelet

Dysfunction Are Exacerbated by

Synthetic Colloids in a Canine

Hemorrhagic Shock Model.

Front. Vet. Sci. 7:641.

doi: 10.3389/fvets.2020.00641 mean ( $95 \%$ confidence interval) was slightly incorrect due to a data entry error. The corrected Table 5 appears below.

In the original article, there was an error. A conversion factor was inadvertently omitted from the equation for thrombodynamic potential index (TPI).

A correction has been made to Materials and Methods, Coagulation Parameters, Paragraph 3:

Rotational thromboelastometry (ROTEM ${ }^{\circledR}$ delta, Tem International GmbH) was performed according to the manufacturer's instructions and PROVETS guidelines (28) using the INTEM (star-TEM and in-TEM reagents), EXTEM (star-TEM and $r$ ex-TEM reagents), FIBTEM ( $\mathrm{r}$ ex-TEM and fib-TEM reagents), and APTEM ( $\mathrm{r}$ ex-TEM and ap-TEM reagents) profiles. Measurement was started $30 \mathrm{~min}$ after sample collection. Each profile was run for at least $1 \mathrm{~h}$ following initiation. Data recorded for the INTEM and EXTEM profiles included clotting time (CT), clot formation time (CFT), alpha angle $(\alpha)$, maximum clot firmness (MCF), and lysis index at $60 \mathrm{~min}$ (LI60). Thrombodynamic potential index (TPI) was recorded for the EXTEM profile as a measure of global coagulation (29), calculated using the equations:

$$
\begin{aligned}
\mathrm{TPI} & =\mathrm{EMX} * 30 / \mathrm{CFT}, \\
\mathrm{EMX} & =(100 * \mathrm{MCF}) /(100-\mathrm{MCF}) .
\end{aligned}
$$

The authors apologize for this error and state that this does not change the scientific conclusions of the article in any way. The original article has been updated.

Copyright $\odot 2020$ Boyd, Claus, Raisis, Hosgood, Sharp and Smart. This is an open-access article distributed under the terms of the Creative Commons Attribution License (CC BY). The use, distribution or reproduction in other forums is permitted, provided the original author(s) and the copyright owner(s) are credited and that the original publication in this journal is cited, in accordance with accepted academic practice. No use, distribution or reproduction is permitted which does not comply with these terms. 
TABLE 2 | Rotational thromboelastometry parameters (mean, 95\% confidence interval) in dogs ( $n=6$ per group) with hemorrhagic shock given $20 \mathrm{~mL} \mathrm{~kg}{ }^{-1}$ of either fresh whole blood (FWB), hydroxyethyl starch 130/0.4 (HES), 4\% succinylated gelatin (GELO), or $80 \mathrm{~mL} \mathrm{~kg}^{-1}$ of balanced isotonic crystalloid (CRYST).

\begin{tabular}{|c|c|c|c|c|}
\hline Parameter & Baseline & Shock & T60 & T180 \\
\hline \multicolumn{5}{|c|}{ INTEM CT (sec) } \\
\hline FWB & $279.3(137.5-421.2)$ & 204.8 (123.1-286.5) & 330.5 (17.2-643.8) & $269.0(150.7-387.3)$ \\
\hline HES & $201.2(129.1-273.2)$ & 145.8 (72.2-219.5) & 231.8 (130.4-333.3) & $199.0(113.0-285.0)$ \\
\hline GELO & 221.5 (146.0-297.0) & 161.0 (117.0-205.0) & $224.3(108.6-340.1)$ & 193.5 (146.5-240.5) \\
\hline CRYST & $143.5(119.1-167.9)$ & 227.8 (190.9-264.8) & $298.8(269.5-328.1)$ & 234.5 (207.6-261.4) \\
\hline HES & 202.8 (106.9-298.8) & 264.0 (168.0-360.0) & 431.5 (287.3-575.7) & $309.2(221.0-397.3)$ \\
\hline GELO & $178.2(128.4-227.9)$ & $186.2(114.2-258.1)$ & 305.5 (211.1-399.9) & 278.7 (178.1-379.2) \\
\hline \multicolumn{5}{|l|}{ INTEM $\alpha\left({ }^{\circ}\right)$} \\
\hline FWB & $54.2(44.9-63.4)$ & $54.0(46.3-61.7)$ & 52.7 (38.5-66.8) & $56.2(49.8-62.6)$ \\
\hline \multicolumn{5}{|c|}{ INTEM MCF (mm) } \\
\hline FWB & $51.2(46.9-55.4)$ & $47.7(43.0-52.3)$ & $45.3(38.3-52.3)^{+}$ & $48.2(44.5-51.8)^{+}$ \\
\hline CRYST & $54.2(50.8-57.5)$ & $46.3(43.0-49.7)$ & $42.2(39.9-44.4)$ & $44.0(41.4-46.6)$ \\
\hline HES & $50.3(45.3-55.4)$ & $44.5(40.3-48.7)$ & $37.0(31.6-42.4)^{\&}$ & $41.0(36.2-45.8)^{\&}$ \\
\hline GELO & $54.0(48.3-59.7)$ & $51.2(45.8-56.5)$ & $41.5(36.0-47.0)$ & $42.8(37.2-48.5)$ \\
\hline \multicolumn{5}{|c|}{ INTEM LI60 (\%) } \\
\hline FWB & $99.0(97.4-100.6)$ & 99.7 (98.8-100.5) & 99.7 (98.8-100.5) & $99.2(97.9-100.4)$ \\
\hline CRYST & $99.7(98.8-100.5)$ & 98.7 (97.6-99.8) & $96.8(94.7-99.0)$ & $97.2(94.4-99.9)$ \\
\hline HES & $99.2(97.9-100.4)$ & $98.5(96.4-100.6)$ & $87.8(67.3-108.4)$ & $90.0(74.1-105.9)$ \\
\hline GELO & $99.5(98.6-100.4)$ & $99.7(99.1-100.2)$ & $98.3(94.0-102.6)$ & 96.5 (87.5-105.5) \\
\hline \multicolumn{5}{|c|}{ EXTEM CT (sec) } \\
\hline CRYST & $129.3(115.4-143.3)$ & $184.2(152.6-215.7)$ & $246.0(198.3-293.7)$ & $221.0(177.7-264.3)$ \\
\hline HES & $187.2(106.0-268.3)$ & $208.0(137.8-278.2)$ & 373.4 (179.9-566.9) & $437.3(0.0-918.6)$ \\
\hline GELO & $139.3(102.6-176.1)$ & $165.0(115.5-214.5)$ & $230.4(167.7-293.1)$ & $263.3(174.5-352.1)$ \\
\hline \multicolumn{5}{|l|}{ EXTEM $\alpha\left(^{\circ}\right)$} \\
\hline FWB & $59.8(53.5-66.1)$ & $60.0(55.9-64.1)$ & $55.8(48.8-62.9)$ & $58.3(54.4-62.3)$ \\
\hline CRYST & $66.5(64.4-68.6)$ & $63.3(60.9-65.8)$ & $59.3(54.2-64.5)$ & 60.8 (59.0-62.6) \\
\hline HES & $61.7(55.1-68.2)$ & $61.8(56.4-67.2)$ & $46.2(28.6-63.8)$ & $53.0(39.8-66.2)$ \\
\hline GELO & $65.7(62.8-68.5)$ & $63.5(59.9-67.1)$ & $49.5(32.7-66.3)$ & $56.0(52.5-59.5)$ \\
\hline \multicolumn{5}{|c|}{ EXTEM MCF (mm) } \\
\hline FWB & $50.5(46.3-54.7)$ & $44.7(39.6-49.7)$ & $45.3(38.6-52.1)^{+}$ & $47.0(43.4-50.6)$ \\
\hline CRYST & $54.7(52.1-57.2)$ & $45.7(42.1-49.3)$ & $39.0(33.3-44.7)$ & $39.3(34.7-44.0)$ \\
\hline HES & $49.3(42.7-55.9)$ & $42.5(33.4-51.6)$ & $31.2(20.5-41.9)^{\&}$ & $37.8(28.6-47.1)$ \\
\hline GELO & $54.3(47.8-60.8)$ & $50.0(43.4-56.6) B$ & $39.0(26.6-51.4)$ & $40.3(31.9-48.8)$ \\
\hline \multicolumn{5}{|l|}{ EXTEM TPI } \\
\hline FWB & $18.8(12.7-25.0)$ & $12.5(6.7-18.3)$ & $12.3(5.6-19.0)$ & $14.0(9.6-18.4)$ \\
\hline CRYST & $28.5(22.8-34.2)$ & $14.3(10.5-18.2)$ & $8.2(5.6-10.8)$ & $9.3(6.1-12.6)$ \\
\hline HES & $20.2(7.4-32.9)$ & $14.2(1.1-27.3)$ & $4.8(2.0-7.6)$ & $8.2(1.4-14.9)$ \\
\hline GELO & $28.8(15.3-42.4)$ & $21.0(10.3-31.7)$ & $11.2(5.3-17.1)$ & $9.5(4.2-14.8)$ \\
\hline
\end{tabular}


TABLE 2 | Continued

\begin{tabular}{lcccc}
\hline Parameter & Baseline & Shock & T60 & T180 \\
\hline EXTEM LI60 (\%) & & & & \\
FWB & $89.3(66.9-111.7)$ & $78.7(48.1-109.3)$ & $90.7(70.7-110.7)$ & $91.0(78.2-103.8)$ \\
CRYST & $99.0(97.9-100.1)$ & $87.7(73.0-102.3)$ & $84.7(52.9-116.5)$ & $78.8(58.5-99.2)$ \\
HES & $98.3(95.5-101.2)$ & $83.3(56.1-110.6)$ & $74.3(36.5-112.2)$ & $84.3(56.2-112.5)$ \\
GELO & $97.5(93.8-101.2)$ & $86.7(65.3-108.1)$ & $75.8(49.2-102.5)$ & $72.5(42.5-102.5)$ \\
FIBTEM MCF (mm) & & & $3(1.5-5.2)$ & \\
FWB & $4.3(3.5-5.2)$ & $3.5(2.4-4.6)$ & $2.8(1.0-4.6)$ & \\
CRYST & $6.0(3.1-8.9)$ & $4.7(1.9-7.5)$ & $0.7(0.0-2.4)$ & $3.2(1.2-5.1)$ \\
HES & $5.0(2.6-7.4)$ & $2.0(0.0-4.9)$ & $2.0(0.2-3.8)$ & $2.2(0.0-5.2)$ \\
GELO & $4.8(3.3-6.4)$ & $3.8(2.3-5.4)$ & & $2.7(1.1-4.2)$ \\
\hline
\end{tabular}

Fluid was delivered directly after Shock time point.

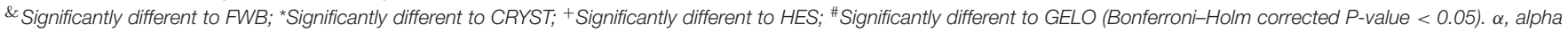
angle; CFT, clot formation time; CT, clotting time; LI60, Iysis index at $60 \mathrm{~min}$; MCF, maximum clot firmness; TPI, thrombodynamic potential index.

TABLE 5 | Platelet closure time and count, rotational thromboelastometry, and plasma coagulation assay parameters (mean, 95\% confidence interval) in dogs $(n=24)$ with hemorrhagic shock.

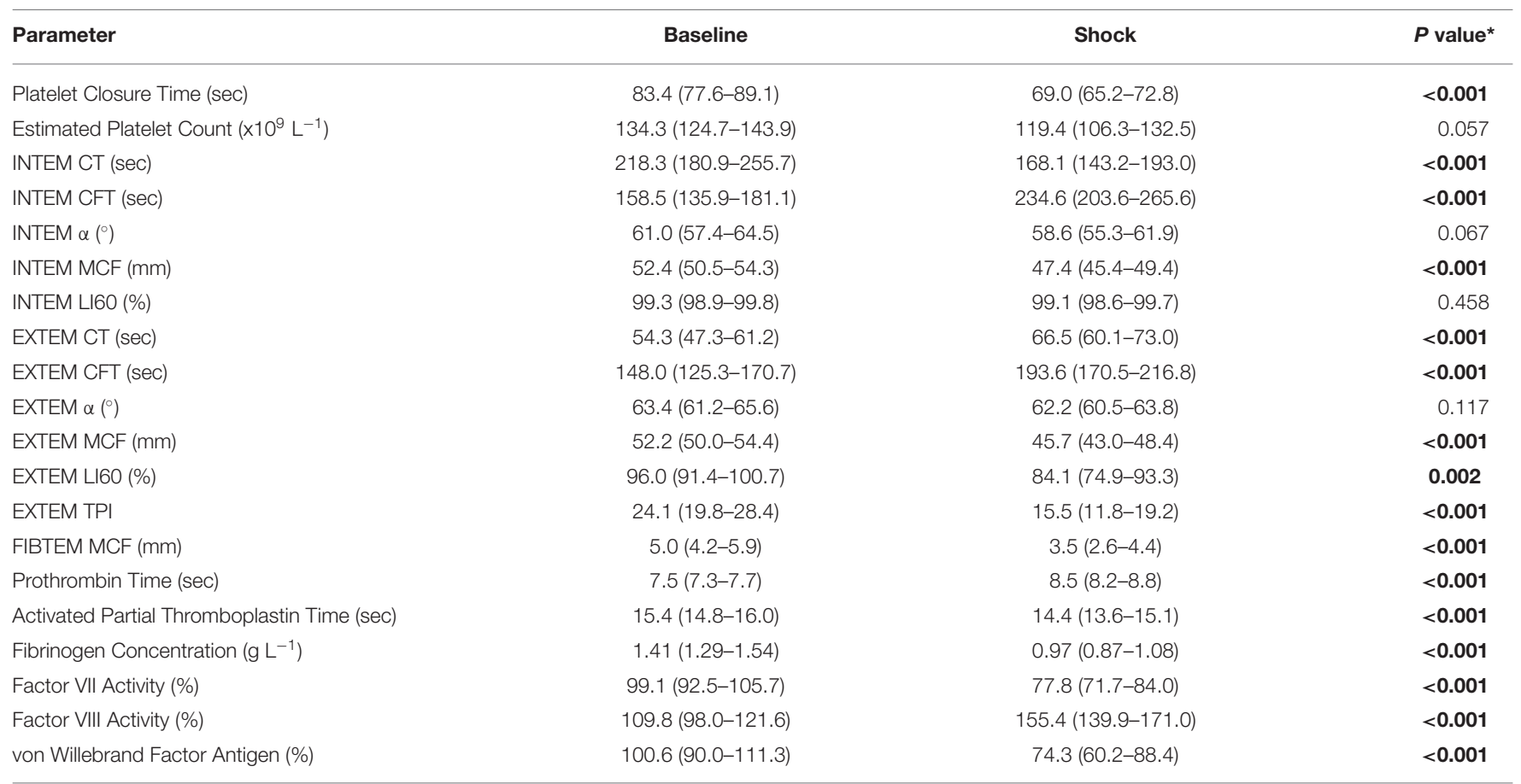

*Paired t-test, bold indicates $P<0.05 . \alpha$, alpha angle; CFT, clot formation time; CT, clotting time; L160, lysis index at 60 min; MCF, maximum clot firmness; TPI, thrombodynamic potential index. 